\title{
NUEVAS METODOLOGÍAS COLABORATIVAS EN LA PRODUCCIÓN DEL ARTE: ENSAMBLAJE CULTURAL EN LA CREACIÓN ARTÍSTICA CONTEMPORÁNEA
}

\author{
Santiago Lara Morcillo \\ Universidad Complutense de Madrid. Doctorando
}

\section{Resumen}

Este artículo expone algunas de las pautas del desarollo de la cultura digital en el marco de la producción artística colectiva. Esta relación ha generado nuevos espacios en red donde se pueden apreciar multitude de plataformas de organización que dependen casi siempre de las necesidades de un colectivo de usuarios determinado. Estas personas formarían parte de un grupo de creadores proactivos que se valen de un procomún como la red para utilizarlo en su propio beneficio y en el del grupo. Estos foros están sujetos al cambio y la experimentación, asímismo también se autorregulan y se transforman atendiendo siempre al desarrollo de la propia comunidad en red. Esto genera nuevas metodologías de producción colaborativa que tienen su reflejo físico en la articulación de espacios de encuentro donde los creadores aúnan su experiencia para crear verdaderos dispositivos de conocimiento entorno al arte.

Palabras-clave: ARTE COLABORATIVO; REDES; PROCOMÚN; ENSAMBLAJE CULTURAL; DISPOSITIVO

\section{NEW COLLABORATIVE METHODOLOGIES IN ART PRODUCTION: CULTURAL ASSEMBLY IN CONTEMPORARY ARTISTIC CREATION}

\section{Abstract}

This article presents some of the patterns in which digital culture is developing within the context of collective artistic production. This relationship has generated new spaces on the web where a multitude of organizational platforms can be appreciated that almost always depend on the needs of a certain group of users. These people would form part of a group of proactive creators who exploit a commons such as the web for their own benefit and that of the group. These forums are subject to change and experimentation, and also self-regulate and mutate, always taking into account the development of their own networked community. This generates new collaborative production methodologies that have their physical reflection in the setting up of meeting spaces where creators combine their experience to create veritable knowledge devices around art.

Keywords: COLLABORATIVE ART; NETWORKS; COMMONS; CULTURAL ASSEMBLY; DEVICE

\footnotetext{
Lara Morcillo, Santiago. "Nuevas metodologías colaborativas en la producción del arte: Ensamblaje cultural en la creación artística contemporánea". AusArt 6 (1): 235-247. D0I: 10.1387/ausart.18901
}

\section{AUSART}




\section{DEL DIY (DO IT YOURSELF) AL DIWO (DO IT WITH OTHERS)}

El desarrollo de la cultura digital ligado a la cultura libre ha llevado a una evolución natural de los procesos de producción y sobre todo a la evolución de la figura del maker (Anderson 2013), cuando se hace referencia a una cierta tradición del Do it yourself (Hazlo tu mismo) que se encontraba en todos los que tenían inquietudes en torno a la cultura del "hacedor", personas a las que les gusta experimentar en el garaje o taller, encerradas, como lo hiciera un científico en su laboratorio. Anderson nos hace una descripción de su abuelo, un ingeniero alemán emigrado a USA que descubrió un novedoso mecanismo de riego para los jardines. Ese gusto por hacer o inventar cosas, le fue transmitido a este autor, quien nos habla sobre los procesos de aprendizaje y los mecanismos creativos que llevaron a ese hombre a estar siempre probando herramientas inventar máquinas. Esta característica es algo básico en la cultura de laboratorio, el "Do it yourself" se ha ido imponiendo más que por una cuestión personal, por una cuestión de necesidad social a lo largo de las últimas décadas. Los procesos creativos en Arte han funcionado de la misma manera, los artistas en su taller han probado, experimentado, reflexionado, interiorizado, etc. en sus estudios de una forma muy parecida a como lo han hecho los ingenieros o los científicos, son procesos muy parecidos. Esta manera de funcionar ha supuesto un paso más para mostrar la evidencia de las capacidades inherentes en todos los individuos, la capacidad de adaptación, aprendizaje y capacidad creativa. El desarrollo de esta metodología ha venido aparejado con el desarrollo de la red y las nuevas relaciones entre individuos. Tanto es así que hoy día sería casi imposible no encontrar algún tutorial en Youtube para resolver cualquier problema técnico. Este desarrollo va unido a la resolución de problemas prácticos y la asimilación de la máxima ensayo-error compartido en la red y fuera de ella. EI DIY, unido a la red y al encuentro de usuarios da lugar al DIWO (Do it with others: Hazlo con otros). Ese taller en el que se pasan horas y horas trabajando en soledad sobre un proceso de creación, ya sea de fabricación o de ideación de un proyecto, pasaría en este caso a convertirse en un espacio donde se insertan varios cuerpos, varias mentes trabajando sobre un mismo proyecto desde perspectivas a veces muy diferentes.

Este proceso se ha ido produciendo en todos los ámbitos del conocimiento y posee dos caras de una misma moneda: por un lado tiene una cara analógica o física: la propia comunidad de usuarios o colaboradores y por otra tiene una cara virtual o digital, ya que a la par de la comunidad física de cuerpos, se genera una comunidad en red muy interesante. Éste grupo organiza y utiliza 
las herramientas de colaboración en red puestas a disposición por la propia comunidad o por otras comunidades. Esta comunidad on-line es polivalente, ya que puede estar formada por los usurarios que trabajan en un proyecto o por otros grupos de usuarios de procedencias geográficas muy distintas. Éstos usuarios anexos, que pueden entrar y salir de dicha comunidad en red, se agregan por una necesidad propia, bien sea porque están trabajando en un proyecto parecido, porque haya alguna implicación directa o indirecta con el proyecto o sencillamente por el hecho de participar y compartir experiencias y conocimiento. Esta característica de doble cara (física y virtual) genera un flujo circulatorio de conocimiento muy importante entre el conocimiento en la red y el hecho físico de compartir la experiencia en un espacio concreto. Por tanto, es un hecho sumamente interesante la relación entre lo local y lo global a la hora de abordar un proyecto artístico de este tipo. Jacques Rancière (2010) nos habla de la nueva topografía de lo posible para referirse a un horizonte que todavía es difuso, pero que supone un campo abonado para la investigación y el desarrollo teórico-práctico de los nuevos procesos de aprendizaje colectivo. El anális de este artículo se basa en el funcionamiento de un proceso de trabajo que se encuentra en esa cartografía. Surgen tres conceptos a tener en cuenta durante el desarrollo de una comunidad colegiada que trabaja en proyectos colaborativos:

a. La articulación de contenidos y de situaciones.

b. La mediación. En todos los individuos se da esta característica, sobre todo en los que provienen de las Humanidades o el Arte. Surge a partir de este tema una reflexión muy interesante en cuanto a la labor de los artistas, que pasarían a ser mediadores dentro de estos equipos de colaboración colegiados.

c. Debido al carácter técnico fuertemente marcado dentro de estos grupos (sobre todo en cuanto al desarrollo del software para llevar a cabo ciertos proyectos). Surge dentro de estos equipos una figura determinante que a veces tiende a condicionar la propia actividad del colectivo. Estos agentes podrían denominarse conseguidores. Estas figuras que son las que manejan el código fuente en la mayoría de los proyectos suelen ser ingenieros de sistemas o profesionales del software que han de estar en diálogo constante con el resto de la comunidad. Hay que destacar que la creatividad en los proyectos artísticos de este tipo fluye de una manera muy peculiar: los artistas o creadores no podemos proyectar la creatividad de una forma unidireccional, sino de una forma circular. Este flujo de creatividad pasa por todos los miembros de la comunidad, y es en las líneas de código 
donde se proyecta gran parte de esta creatividad. Por eso la figura del programador es muy importante, ya que las líneas de código son muy sensibles a los cambios, todo lo que poseen de abstracción lo poseen también de permeabilidad y ductilidad. Es decir, que a veces para un mismo problema hay soluciones diferentes (con líneas de código de programación diferentes). Este proceso de colectivización en la ejecución de proyectos artísticos son producciones culturales colectivas que respetan las diferencias. Por esto mismo aparece una metodología muy interesante alrededor de estos grupos: se trata del ensamblaje cultural. A esto se une otro aspecto fundamental del desarrollo de la nueva sociedad del conocimiento: la cultura 2.0 o web 2.0. Es innegable que el mundo del arte, los procesos de producción y sobre todo el aprendizaje han de pasar por las nuevas herramientas que están conformando individuos diferentes, donde lo que importa es compartir la experiencia y desarrollar nuevas fórmulas de criticidad colectiva.

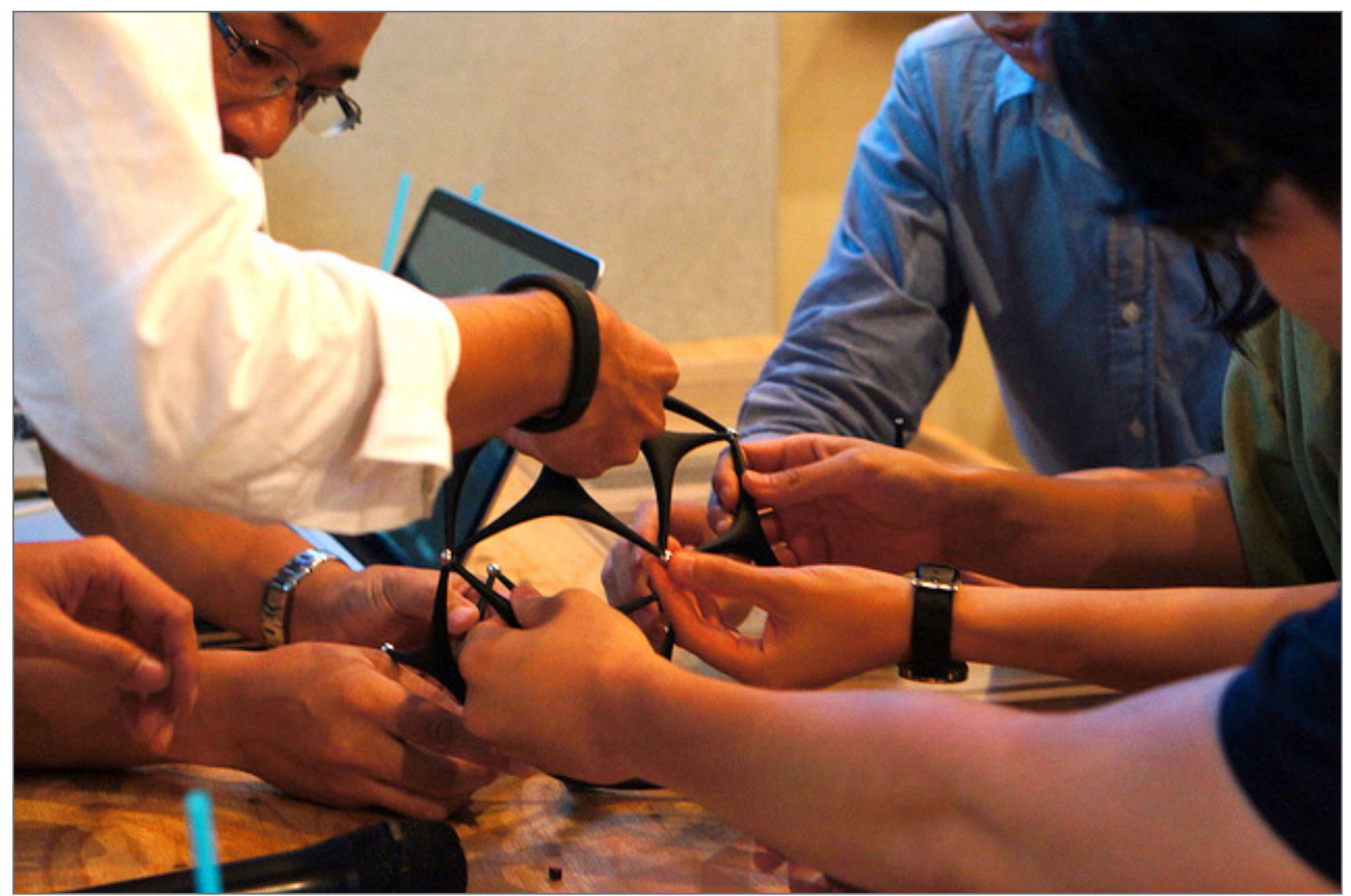

Figura 1. Grupo de trabajo de DIWOLab en el FabCafe de Tokio.

Fuente: http://fabcafe.com/tokyo/blog/a629db0f491b7b886e9e603da92b83b71 
Otra discusión que se abre es el tema de la autoría del artista que ahora pasaría a ser un productor de conocimiento, perdiendo el carácter "chamánico" o "iluminado" al plantear el trabajo de forma colaborativa. Sin embargo es aquí cuando cobra más sentido su función como creador, integrado en un equipo de producción. En este sentido cabe destacar el gran reto que supone para los artistas exportar de alguna manera la imaginación a otros ámbitos del conocimiento. Una última discusión a debatir a partir del análisis de este tipo de metodologías de trabajo sería la búsqueda de otros modelos de economías que focalizarían el problema en el acceso a la cultura y no tanto en el comercio: una cuestión clave para acercar poco a poco la producción cultural a la esfera pública. Todas estas cuestiones nos llevan a analizar los procesos organizativos y las posibilidades materiales para llevar a cabo cualquier proyecto con estas metodologías.

\section{AUTORREGULACIÓN DE LAS COMUNIDADES EN RED}

Una de las dudas que siempre han asaltado a los escépticos del desarrollo de las posibilidades de la red, ha sido la verosimilitud de los contenidos o la fiabilidad de los mismos en torno a un determinado tema. Esta reacción es suscitada a veces por los que no están "alfabetizados digitalmente" o están en proceso de ello. El desarrollo de los bloggers en un primer momento y posteriormente de las redes sociales y apps, han ido calando en la cotidianidad de la población. Estas plataformas se han hecho un hueco en las posibilidades comunicativas a nivel personal y también en la investigación profesional. Estos procesos de interiorización de herramientas digitales por parte de la comunidad de usuarios y su rápida accesibilidad, provocan oleadas de criticidad concentrada y colectiva. Cuando hay un contenido que no es veraz y es colgado en la red por error o mala fe, provoca un múltiple rechazo, ya que es contrastado de forma colectiva y examinado de una manera espontánea por cientos de usuarios. La honestidad, así como la responsabilidad son cuestiones que provocan una limpieza natural de la red, mutando de forma exponencial y generativa por la colectividad, un ejemplo de esto lo encontramos en la Wikipedia. "El ciberespacio exige una comprensión novedosa de cómo funciona la regulación y así, nos compele a mirar más allá del ámbito tradicional de los abogados -más allá de las leyes o incluso de las normas-. El ciberespacio requiere una concepción más allá de la regulación y, lo que es más 
importante, el reconocimiento de un regulador de singular relevancia. Dicho regulador es la oscuridad que da título a este libro-el código. En el espacio real, somos capaces de reconocer de qué modo reglamentan las leyes -por medio de constituciones, estatutos y demás códigos legales. En el ciberespacio, hemos de comprender cómo regula un "código" diferente- esto es, cómo el software y el hardware, que hacen del ciberespacio lo que es, constituyen su código (...) Un código diferente determina una regulabilidad diferente de las redes. La regulabilidad se halla, por tanto, en función del diseño (...) Cuando el comercio escribe el código, ese código puede ser controlado, puesto que las entidades comerciales también pueden ser controladas" (Lessing 2009, 134).

En las comunidades que genera la red todavía existe cierta libertad que al sistema le es difícil controlar. Los usuarios que realizan proyectos artísticos de forma colectiva o que se valen de los foros de conocimiento on-line para su ejecución, han emulado herramientas digitales desarrolladas en un primer momento para la toma de decisiones a nivel político. En ocasiones para que esta democratización sea viable al $100 \%$ surge el consenso aproximado o consenso práctico. Este término aparece en contraposición al consenso por unanimidad que se llevó a cabo en las asambleas del 15M en las plazas de toda España, donde se puso en práctica un modelo "cadena", que por definición es un modelo anti-red. A pesar de que el activismo que se venía desarrollando en la red saltó a la calle de una forma masiva con las acampadas realizadas en 2011, es curioso comprobar como existe una contraposición entre el modelo de asamblea frente al modelo de red-laboratorio. Éste modelo es algo que han venido trabajando los hackers durante mucho tiempo. Esta comunidad ha explorado muchísimo sobre la horizontalidad en la toma de decisiones y la ejecución de propuestas, tanto es así que en ocasiones han llegado a adoptar fórmulas que no son totalmente horizontales simplemente por una cuestión de eficacia. En estas comunidades existe la meritocracia y los denominados "ancianos de la tribu": personas con experiencia que renuevan su aceptación mediante su aportación constante y contrastada por la propia comunidad. En muchas ocasiones la horizontalidad llevada a sus últimas consecuencias ralentiza y dificulta la realización de los proyectos. En este sentido la meritocracia y la democracia se entrelazan, potenciado la autorregulación y la autocensura dentro del colectivo. La red, por tanto, es el operador de posibilidad, las diferentes plataformas y espacios en Internet donde se potencian o desechan los resultados nos lleva la mirada hacia esas transformaciones. Internet aparece como algo vivo en transformación constante, los usuarios lo construyen dependiendo de las necesidades del momento. 


\section{PROSUMIDORES: NUEVOS DISPOSITIVOS CULTURALES DE COALICIÓN}

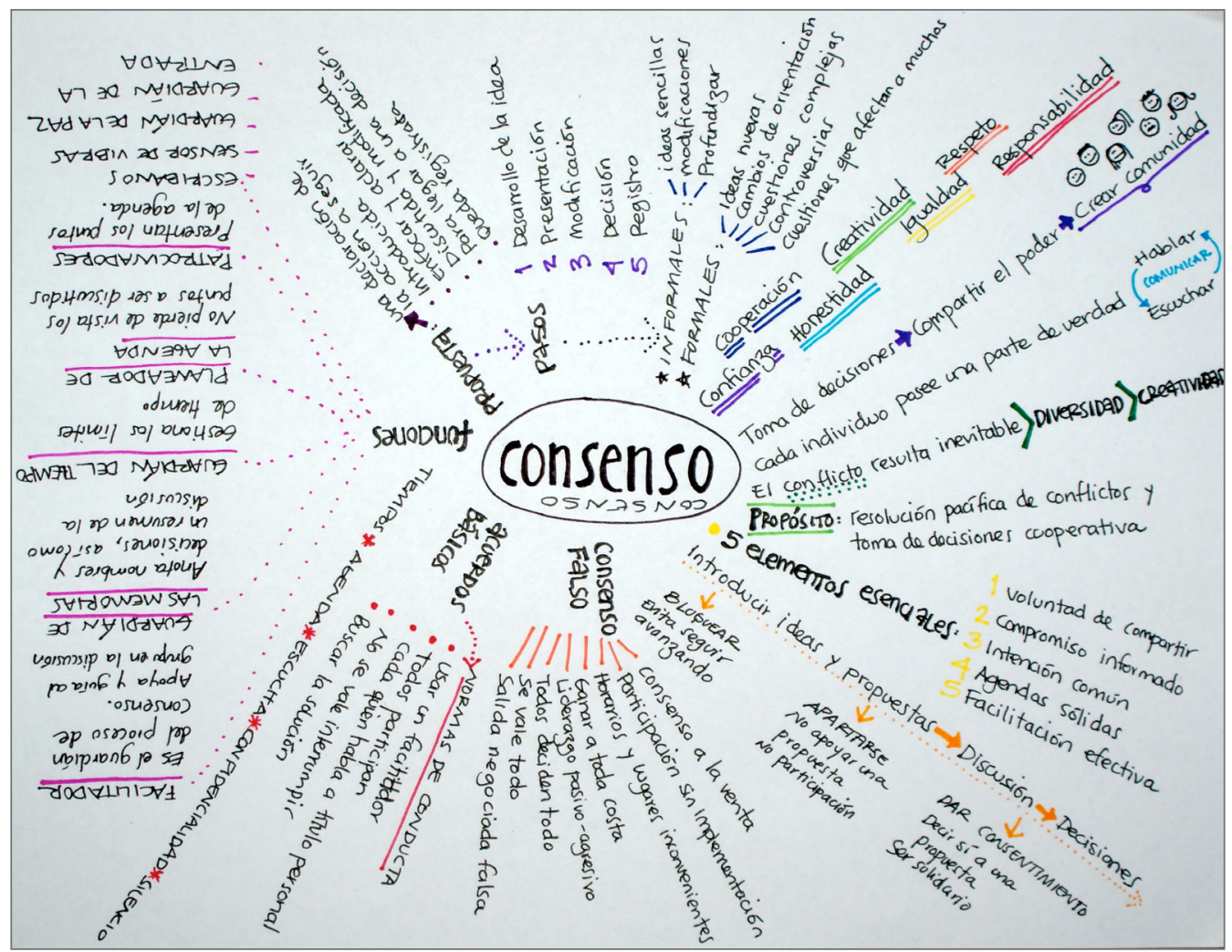

Figura 2. Mapa conceptual

Esta nueva necesidad de horizontalidad en la cultura y en el ámbito del pensamiento sería necesaria también para el arte. Quizá estos nuevos planteamientos son herederos del arte contextual (Ardenne 2006), lo cierto es que esta nueva estructura horizontal afecta a todos los ámbitos del pensamiento. Estos referentes afectan al aprendizaje, a la creación y a la difusión de la información. El procedimiento en este ámbito horizontal es empático y lo que importa es, sobre todo, compartir experiencias. El desarrollo de la red ha propiciado aún más este gusto por compartir información. Tenemos como claro ejemplo en la cultura digital el software libre y el copyleft. Fruto de esta cultura Commons o Procomún esta experiencia directa es inmediata y en ocasiones transgrede los parámetros impuestos por el poder económico. El desarrollo 
del trabajo en red en centros tipo laboratorio (medialabs, fablabs, makespaces, etc.) y su evolución e importación a otros nodos de conocimiento, implica también un posicionamiento político de base, no tanto ideológico, sino estructural. Lo que se pone en tela de juicio con estos nuevos métodos de trabajo es el carácter jerárquico que tienen la mayoría de nuestras instituciones y cómo poco a poco podrían transformarse en núcleos horizontales que faciliten el acceso a la comunicación y al progreso. Se torna necesaria una "repolitización de la esfera pública que condujese a la gobernanza de los asuntos de interés común de la ciudadanía. Sólo entonces, y ahí podríamos hablar de la constitución de una cierta e-utopía -cuya contribución pudiera apelar al establecimiento de una cierta democracia directa electrónica" (Brea 2010, 112). Estas nuevas y necesarias estrategias ciudadanas entroncan directamente con el concepto de táctica o maniobra (Certeau 2000): indagando en el interior de las estructuras, los ciudadanos se apropian del espacio organizado y cambian su programación.

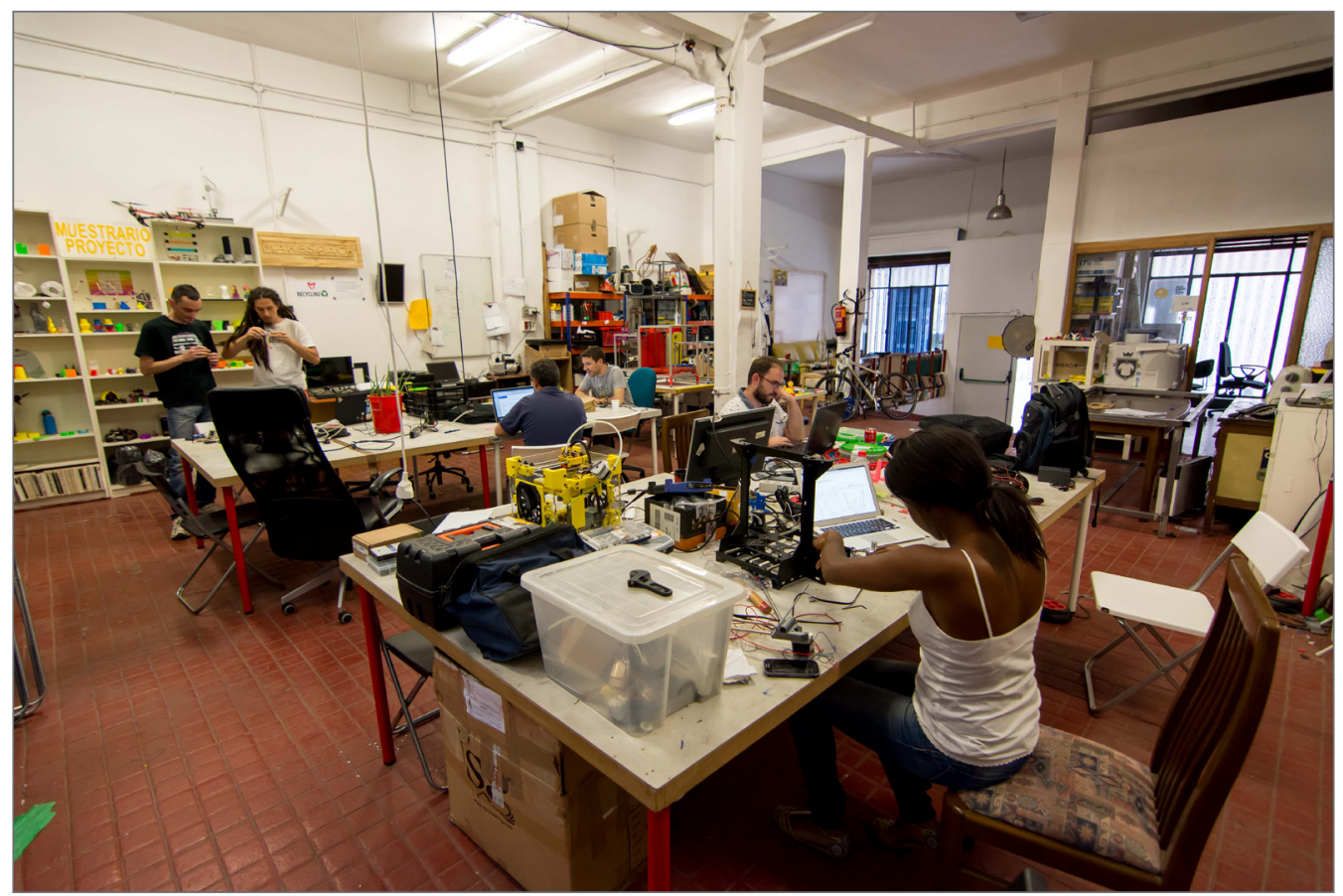

Figura 3. Interior del Makespace Madrid.

En una sociedad en constante contracción en cuanto a los derechos sociales conquistados por generaciones anteriores, la auto-organización por parte de la ciudadanía para recuperarlos se hace necesaria. Esto se ha conver- 
tido estos últimos años en un hecho irrefutable, desde la aparición del 15M, pasando el surgimiento de las diferentes mareas sociales ("marea blanca" en sanidad; "marea verde" en educación, etc.). Estos síntomas han sido la consecuencia de un cambio de estructura en la forma de relacionarnos a nivel cotidiano, pasando desde la intimidad hacia lo local, para proyectarse hacia lo global como un todo, como un cuerpo colectivo que está reinventando la política para recuperar ciertos derechos que se nos han ido arrebatando en estos últimos años. La reinvención de los derechos sociales ha generado un nuevo mapa político. "La nueva izquierda sólo es imaginable en términos de producción de subjetividad para la producción de libertad; únicamente es imaginable como sujeto de gestión de lo común, no ya gestión del poder sino de lo común, al cual se subordina el poder. Esto es fundamental, se trata de reconocer que la realidad en la que vivimos es un dato absolutamente común y que nuestras singularidades se mueven en esta comunidad. Existimos como singularidades en la relación que se produce dentro de lo común. Somos individuos y realidades que viven y se expresan en cohesión entre estas singularidades. Común es la relación en la que nos ponemos unos con otros. Y esta relación es productiva; lo común es una producción que las singularidades expresan como lenguaje, como subjetividad y como vida en común, en una dimensión absolutamente biopolítica" (Negri 2007, 112). La articulación y generación de dispositivos por parte de la ciudadanía se han hecho necesarias, no sólo para recuperar ciertos aspectos democráticos en nuestras sociedades contemporáneas, sino porque estos dispositivos cubren parte de las necesidades que no están siendo cubiertas debido a la ruptura del contrato social por parte de las grandes élites financieras. Por tanto, a partir de aquí constataríamos que "el poder colectivo de los cuerpos en el espacio público es todavía el instrumento más eficaz de oposición cuando todos los demás medios de acceso quedan bloqueados" (Harvey 2013, 232). Esa creatividad difusa y dispersa en la sociedad es la que "debe unir a artistas y trabajadores creativos cuyos talentos se convierten tan a menudo en productos comerciales bajo el control de las grandes fortunas" (ibídem). La cultura en general es muy sensible a cualquier cambio social que se produzca, por lo que desde este paisaje, las necesidades están bastante identificadas. Las instituciones, concretamente los museos o centros de arte que han ido apareciendo a lo largo de los años de bonanza económica y que posteriormente con la crisis han tenido que reinventarse y ejecutar sus proyectos con presupuestos notablemente adelgazados, no están cubriendo las necesidades de lo local. Estas necesidades culturales no pasan por grandes exposiciones, se hacen necesarios lugares de encuentro, producción y experimentación, donde los usuarios "consumidores" de cultura o arte, pasen a ser usuarios pro-activos (prosumidores) dentro de los espacios. El carácter divulgativo de los museos no ha de centrarse tanto el tema expositivo. 
Lo que la gente está demandando es una cierta horizontalidad en las instituciones que gestionan lo simbólico y no tanto proyectos donde las programaciones se cierran de una forma vertical y sin participación ciudadana alguna.

\begin{tabular}{|c|c|c|}
\hline PROSUMIDOR DE ARTE & VS. & CONSUMIDOR DE ARTE \\
\hline Productor de procomún & & Espectador en la Industria Cultural \\
\hline Pro-activo & & Pasivo \\
\hline
\end{tabular}

Frente a este fenómeno han surgido estos últimos años cantidad de espacios alternativos donde han aparecido modelos nuevos de gestión entorno a lo artístico. Se han desarrollado diferentes dispositivos de conocimiento por parte de la ciudadanía a veces instaurados en la institución y otras fuera de ella. Estas formas de operar suponen nuevos dispositivos culturales, que proyectan nuevos modos de relación entre individuos que conforman un entorno cultural. Estos grupos surgen por la necesidad. Los cambios y transformaciones que están teniendo lugar en nuestra sociedad van encaminados a una demanda creciente de derechos por parte de los ciudadanos, que cada vez son más conscientes de que las micropolíticas de lo local son más importantes para poder tener acceso a un mejor desarrollo individual y colectivo. En cuanto al ámbito de la cultura se vislumbraría aquí una configuración y materialización de un espacio concreto de actuación en el marco del tercer umbral (Brea 2004) en una amplia cartografía rápida de la evolución del arte contemporáneo, en relación con sus horizontes de contraste, los modos dominantes de la producción y el ámbito de reclamo ciudadano de derechos. A raíz de esta necesidad surgen casi de forma espontánea las nuevas redes relacionales, nuevos grupos de actuación ligados a la institución o independientes, que generan foros de conocimiento y nodos de actividad cultural alternativa cada vez más importantes en nuestra sociedad. Medialab-Prado o Makespace Madrid son ejemplos de ello, uno actuando desde dentro de la institución (Medialab Prado depende del Ayuntamiento de Madrid) y el otro desde fuera de ella. Estos espacios están ligados al desarrollo de nuevos dispositivos mediales (Brea 2002), en clara referencia a al desarrollo de los dispositivos como herramientas y técnicas que incrementan el desarrollo de la cultura digital. Será en "Las tres eras de la imagen" donde José Luis Brea usa el término "coalición" para referirse a la "articulación gerenciada" por el modelo de las redes sociales y la web 2.0, unida a un trabajo de transformación en las condiciones de producción. Nos habla de "una comunidad de comunicación que pudiera efectivamente estructurarse como genuina de 'productores de medios' -la expresión 
bien conocida, es de Brecht y en la que como tal, la totalidad de los receptores pudiera efectivamente a la vez, y en las mismas condiciones que el resto, constituirse en emisor - una comunidad, por tanto de participadores en la que la comunicación y las transferencias de imaginario no fluyera más nunca de uno -o pocos- a muchos, sino de todos a todos, según el programa de la referida democracia extendida, acaso directa" (Brea 2010, 112). Estos dispositivos de conocimiento utilizados en la ciencia y la cultura crecen y se nutren mediante la experimentación, no podíamos dejar de analizar dicho concepto, pues en esa evolución técnica están inmersas la mayoría de los procesos digitales en la actualidad, influyendo en los modos y desarrollos culturales, actualmente en período de pruebas.

\section{CONCLUSIONES}

Una de las características esenciales que los creadores adoptan a la hora de experimentar estas nuevas estrategias y metodologías digitales, es la adopción de un posicionamiento de mediación. Esta postura en ocasiones forma parte de una estrategia de aprendizaje personal, una alfabetización digital que se produce en varias fases y que no solo trata de arte realizado de forma colaborativa, sino de los cambios de concepción sobre el propio sistema del arte actual. A partir de esta mediación se amplía la figura del creador como generador de conocimiento: aparece una articulación de la creatividad dispersa que se encuentra en el ámbito digital, en la creación de código informático, en los diferentes usos de herramientas digitales o en la propia forma de trabajo colaborativo. El artista se convierte en un especialista dentro de un entorno de producción colegiado, ampliando su propio espectro profesional al incursionar en otros lenguajes creativos vinculados casi siempre al software. La cuestión de la autoría se matiza, ya que los creadores que operan de esta forma no obedecen tanto a una concepción romántica del artista, sino a un perfil más actualizado que se nutre de un posicionamiento mediador. Otro aspecto afectado es lo económico, ya que los nuevos dispositivos de conocimiento colectivo promueven una visión más allá del aspecto comercial, proyectando la necesidad de hacer pública la experiencia artística en relación a lo digital. Se generan por tanto, grandes interrogantes que abren un campo de experimentación con las economías híbridas, donde lo privativo y la economía de compartición conviven de muy diversas formas, unas veces nutriéndose con el uso de redes y comunidades (plataformas) de creación colectiva, otras simple- 
mente conviviendo desde la necesidad de proyectarse a una mayor cantidad de público.

Lo empírico aparece aquí como el pilar sobre el que se basan todas estas nuevas metodologías. Mediante la experiencia colectiva se produce una búsqueda de nuevas retóricas. La investigación colaborativa puede partir de diferentes estrategias de articulación, de ensamblaje y de hibridación de elementos materiales, de software o hardware, electrónicos, conceptuales, etc. que se nutren de la observación de otros creadores que se mueven en los mismos términos, donde la tecnología es parte del elemento retórico de la obra. El uso metafórico de lo digital juega aquí un papel fundamental. Sobre el tipo de obras que se producen mediante estas metodologías hay que decir que se tratan en su mayoría de obras de arte abiertas: prototipos generados a partir de la cultura libre, con múltiples posibilidades tanto metodológicas como de mejora. Al depender éstas de elementos electrónicos cambiantes, hay que jugar con la obsolescencia de la tecnología digital, que es exponencial a lo largo del tiempo. Son las obras de prototipado las que pueden aportar soluciones a todas estas cuestiones con múltiples aplicaciones y direccionalidades. Así lo que hoy se reproduce en una pantalla mp3, mañana será reproducido en una tablet o un smartphone, lo que ayer se reproducía con un proyector convencional hoy se proyectará con un proyector LED o un pico-proyector. Esto conlleva a una concepción híbrida de las obras, así como de la propia cultura en general.

\section{Referencias}

Anderson, Chris. 2013. Makers, la nueva revolución industrial. Traducción, Javier Fernández de Castro.Barcelona: Empresa Activa

Ardenne, Paul. 2006. Un arte contextual: Creación artística en medio urbano, en situación, de intervención, de participación. Traducción, Françoise Mallier. Murcia: Cendeac

Brea, José Luis. 2002. La era postmedia: acción comunicativa, prácticas (post)artísticas y dispositivos neomediales. Salamanca: Consorcio Salamanca

- . 2004. El tercer umbral: Estatuto de las prácticas artísticas en la era del capitalismo cultural. Murcia: Cendeac

— . 2010. "E-Utopías". En: Las tres eras de la imagen: Imagen-materia, film, e-image. Madrid: Akal

Certeau, Michel de. 2000. La invención de lo cotidiano. I. Artes de Hacer. Traducción de Alejandro Pescador. México DF: Universidad Iberoamericana

Harvey, David. 2013."\#OWS [Occupy Wall Street]: El partido de Wall Street se topa con su némesis". En Ciudades rebeldes: Del derecho de la ciudad a la revolución urbana, Traducción de Juanmari Madariaga. Madrid: Akal 
Lessing, Lawrence. 2009. El código 2.0. Edición e introducción Florentino Cabello. Madrid: Traficantes de Sueños

Negri, Antonio. 2007. "La comuna de Madrid". En: Goodbye Mr. Socialism: La crisis de la izquierda y los nuevos movimientos revolucionario, conversaciones con Raf Valvola Scelsi; traducción de Rosa Rius Gatell y Carme Castells Auleda. Barcelona: Paidós

Rancière, Jacques. 2010. El espectador emancipado. Traducción de Ariel Dilon; revisión de Javier Bassas Vila. Castellón: Ellago 